\title{
How does benefit finding evolve over time among Chinese women breast cancer survivals: a longitudinal study
}

\section{Weiyun Bi ( $\sim$ bwy1001@126.com )}

the first affiliated hospital of the Air Force Military Medical University

\section{Huaning Wang}

the first affiliated hospital of the Air Force Military Medical University

Guitao Yang

the first affiliated hospital of the Air Force Military Medical University

Cailin Zhu

First Affiliated Hospital of Xi'an Jiaotong University

\section{Research Article}

Keywords: breast cancer, benefit finding, post-traumatic growth

Posted Date: April 28th, 2021

DOl: https://doi.org/10.21203/rs.3.rs-340763/v2

License: (a) (1) This work is licensed under a Creative Commons Attribution 4.0 International License.

Read Full License 


\title{
How does benefit finding evolve over time among Chinese women breast cancer survivals: a longitudinal study
}

\author{
Weiyun $\mathrm{Bi}^{\mathrm{a}, *}$, Huaning Wang ${ }^{\mathrm{b}, *}$, Guitao Yang ${ }^{\mathrm{a}}$, Cailin $\mathrm{Zhu}^{\mathrm{c}, * *}$ \\ ${ }^{a}$ Clinical Skills Training Center, the first affiliated hospital of the Air Force Military Medical University, Xi'an, PR China \\ ${ }^{b}$ Department of Psychosomatics, the first affiliated hospital of the Air Force Military Medical University, Xi'an, PR China \\ ${ }^{c}$ Department of Surgery, the first affiliated hospital of Xi'an Jiaotong University, Xi'an, PR China,
}

\begin{abstract}
Objective: Even though the prevalence of benefit finding (BF) has been empirically shown to exist among breast cancer (BC) survivals, how does benefit finding evolve over time remains inadequately investigated. This objective of this study is to examine how BF evolves over time among Chinese breast cancer survivals and determine the demographic, medical and psychosocial factors that can sustain BF increase over time, thus bring about higher level of long-term post-traumatic growth among breast cancer survivals.
\end{abstract}

Methods: Participants were 486 women with different stages of breast cancer (stages I, II and III) followed from completion of primary treatment. Analysis were performed on the data collected during 2014-2019. During the assessment, each participant completed self-report questionnaires of characteristics and benefit finding at six time points with the interval of six months since $\mathrm{BC}$ diagnosis. The relationships between demographic, medical and psychosocial characteristics and benefit finding evolution over time were examined using mixed models.

Results: Participants reported mixed results on the evolving patterns of benefit finding: $28 \%$ reported an upward trend in BF scoring over time, $49 \%$ instead reported an downward trend, and the remaining $23 \%$ reported no obvious change. Our study has shown that some well-known covariates of benefit finding, e.g. education, income, and social support, are not associated with BF trends. In comparison, levels of spirituality and disease coping at diagnosis can more reliably predict BF evolution over time. Our results strongly indicate that benefit finding can be sustained and increased by encouraging attempts at meaning-making and active disease coping during breast cancer treatment. To our knowledge, this study is among the first to examine trends of benefit finding evolution over time on breast cancer survivals and determine their psychosocial predictors in developing countries.

Conclusion: Identifying the sustaining factors of benefit finding in the experience of breast cancer is the key to design psycho clinical solutions for patients' long-term post-traumatic growth. As time goes by, cancer patients may experience less benefit finding. However, active disease coping and meaning-making by patients can effectively counter this undesired trend and boost their higher long-term post-traumatic growth.

Keywords: breast cancer, benefit finding, post-traumatic growth.

\section{Introduction}

Breast cancer (BC) is one of the most common malignant diseases worldwide. As prognosis of $\mathrm{BC}$ has improved over the last years, research on quality of life of BC survivors has become increasingly important. Many studies have shown that a high percentage of BC survivors reported positive changes in their experience of disease [1, 2]. Benefit finding has been proposed as one of two major constructs of positive consequences of cancer, which also include

\footnotetext{
*Weiyun $\mathrm{Bi}$ and Huaning Wang contribute equally to the work and should be considered as co-first authors.

${ }^{* *}$ Corresponding author

Email address: zhuclin@xjtufh.edu.cn (Cailin Zhu)
} 
post-traumatic growth (PTG) [3, 4, 5]. BF was defined as the process in which a patient re-assigns positive value to his (or her) illness based on the benefits he (or she) identifies. In contrast, PTG refers to the more general positive changes in life perspective, interpersonal relationship and self-perception. While BF is supposed to start immediately after diagnosis, PTG, referring to the active change in one's capacity to deal with adverse events, may develop even years after diagnosis [6, 7, 8].

Benefit finding has been frequently examined in the context of breast cancer survivorship. Early investigations of $\mathrm{BC}$ benefit finding have demonstrated that most women reported positive life changes due to their diagnosis [9]. To date, the existing studies on BC benefit finding mainly investigated the covariates of benefit finding in various demographic groups [10, 11, 12, 13]. They showed that greater benefit finding may be associated with miscellaneous factors, e.g. education level, spirituality, illness intrusiveness, social support and mental health. However, even though benefit finding is typically assumed to increase over time following cancer diagnosis [14], as increased time allows for additional cognitive processing of the diagnosis, preliminary findings in the literature are actually mixed. Some studies reported an increase in benefit finding over time after cancer diagnosis [4], while others instead reported a decrease [15, 16]. It is noteworthy that the primary objective of the existing studies was to identify the covariates of benefit finding. The issue of $\mathrm{BF}$ evolution over time unfortunately remains inadequately investigated.

However, the issue of how BF evolves over time in breast cancer survivals is critically important given that BF and PTG are closely correlated [17, 18]. In other words, increasing BF after diagnosis can effectively boost longer-term PTG of cancer survivals, thus improve their quality of life. The aim of the present study is twofold: (a) to examine the trends of BF evolution over time among breast cancer survivals; and (b) to identify the factors that can sustain $\mathrm{BF}$ increase over time. We hypothesize that BC survivors would generally report some extent of benefit finding after diagnosis, but whether BF can be sustained over time and develop into longer-term PTG depends on some potential psycho and social factors, which include age, education level, income, social support, spirituality and active disease coping.

\section{Methods}

\subsection{Participants}

This observational, longitudinal study was conducted with 486 women aged between 20 and 50 years and diagnosed with stage I-III breast cancer between 2014-2017. Women were recruited through the first affiliated hospital of the Air Force Military Medical University and screened by chart review.

Each eligible women were emailed a baseline questionnaire to complete and return response. Baseline questionnaires were completed within 6 months of diagnosis. Follow-up questionnaires were completed 6, 12, 18, 24, and 30 months after baseline. Because the baseline questionnaire was administered to women at differing lengths of time following diagnosis, we used the dates of completion of each survey along with the date of diagnosis to create a continuous variable of time since diagnosis (in months).

Of 1028 eligible women in two hospitals, 667 consented to participate. In appreciation of their time, enrolled participants could select from several promotional items ( $<100 \mathrm{RMB}$ value). Due to cancer recurrence or lost contact (moving out of Xi'an), some of the initial participants dropped out during our study. During the assessment period, 637 participants completed the 1st-year assessment, 579 completed the first 2-year assessment, and 486 completed the entire 3-year assessment. Given our focus on benefit finding over time, we limited analysis to the 486 participants who had completed the entire 3-year assessment and were still disease free at the end of assessment.

\subsection{Measures}

We focus on the demographic, medical and psychosocial characteristics, which may to some extent affect benefit finding.

\subsubsection{Demographic characteristics}

Participants reported their age, marital status, educational level, income, and employment status.

\subsubsection{Medical factors}

A medical chart review was performed upon completion of primary treatment. Data included the following: cancer stage(I or II or III), type of surgery, receipt of radiation, and receipt of chemotherapy. 


\subsubsection{Social support}

The RAND Social Support Scale measured respondents' evaluation of the resources provided by their social network [19]. It measures four aspects of support: emotional, tangible, affection, and social interaction [20]. A total score sums the four categories with a possible total score ranging from 20 to 100 (higher scores means greater support).

\subsubsection{Spirituality}

Spirituality was assessed by the Functional Assessment of Chronic Illness Therapy - Spiritual Well-Being (FACITSp) scale [21, 22]. This 12-item scale contains two subscales: meaning/peace and faith. Since the participants in our study are mostly Han Chinese, who usually have no or very weak religion, we mainly measured spirituality scoring on the meaning/peace subscale. The eight-item meaning/peace subscale assesses a sense of meaning, peace, and purpose in life (Cronbach's $\alpha=0.83$ ). Higher scores indicate greater spiritual well-being.

\subsubsection{Disease coping}

Disease coping was assessed using the Brief COPE [23]. The subscales from the Brief COPE include active coping, planning, acceptance, instrumental and emotional social support seeking ( 2 items each). Items were completed in reference to "what you have been doing to cope with your experience of cancer, including your current physical or emotional concerns related to your cancer experience" and were rated on 4-point scale ( $1=\mathrm{I}$ don't do this at all; $4=\mathrm{I}$ do this a lot). The means of each subscale were used to compute an overall composite mean, giving each subscale equal weight in the composite. Not surprisingly, subscales were positively and significantly correlated ( $\mathrm{r}=0.15$ to 0.71 , $\left.\mathrm{p}_{i} 0.05\right)$. It is noteworthy that internal consistency was acceptable in the current study $(\alpha=0.79)$.

\subsubsection{Benefit finding}

We measured the Benefit Finding Scale (BFS) by a Chinese version [24], which was translated from the original BFS proposal [25]. The Chinese version consists of totally 19 items and asks participants to respond to "Have you found any positive change from the experience of your cancer" on a scale from 1 (not at all) to 4 (a great deal of change). Possible BF scores vary from 19 to 76. In our study, Cronbach's $\alpha$ for the BFS was 0.86 .

\subsection{Analytical Strategies}

Multivariate linear models were used to assess effects of demographic, medical, and psychosocial variables on BFS scores at baseline, and random coefficient models were used to assess effects of these variables on BFS scores in longitudinal analysis over all time points. A variable is considered correlated with BFS if its $p$-value is less than 0.05.

For trend analysis, time was calculated as multiples of 6-months since diagnosis and included in the model using linear term. For each participant, we estimate its BF trending slope, which is denoted by $\beta$, by a linear regression model. We categorized BFS trend patterns into three trends (upward, downward or no obvious change) according to their estimated $\beta$ values. Specifically, $\beta \geq 0.3$ means upward, $\beta \leq-0.3$ means downward, while $-0.3<\beta<0.3$ means no obvious change. Then, we analyzed the association between various variables and $\mathrm{BF}$ trending slopes (or $\beta$ ) by multivariate linear models. The predictors of BF trends were identified with the $p$-value threshold set at 0.05 .

\section{Results}

\subsection{Covariates of $B F$}

The detailed characteristics of the sample have been presented in Table 1. It is clear that benefit finding was found on the participants after cancer diagnosis. The results of association analysis have been presented in Table 2 It can be observed that at baseline, BF score was significantly associated education level, income level, social support, spirituality, and disease coping $(p \leq 0.05)$. It was however not observed to be related to any other studied social and psycho variable $(p>0.05)$.

The results of longitudinal association analysis have also been shown in Table 2 The results indicated that education, income, social support, spirituality and disease coping are closely correlated with time-varying benefit finding scoring. It can be observed that both social support and use of active coping strategies was highly correlated with changes of BFS over time. A 1-point increase in time-varying social support means a 1.94-point increase on BF 
Table 1: Characteristics of the study sample $(\mathrm{N}=486)$ and $\mathrm{BF}$ scoring at baseline

\begin{tabular}{|c|c|c|}
\hline Demographic/medical characteristics & $\mathbf{N}(\%)$ & BFS-baseline M(SD) \\
\hline \multicolumn{3}{|l|}{ Age (years) } \\
\hline$<30$ & $85(17)$ & $57.35(4.22)$ \\
\hline$\geq 30,<40$ & 284(59) & $55.34(3.14)$ \\
\hline$\geq 40,<50$ & $117(24)$ & $57.45(4.38)$ \\
\hline \multicolumn{3}{|l|}{ Marital status } \\
\hline Married & $414(85)$ & $56.39(2.96)$ \\
\hline Others & $72(15)$ & $55.10(5.68)$ \\
\hline \multicolumn{3}{|l|}{ Education } \\
\hline$\leq$ High school & $223(46)$ & $54.26(3.44)$ \\
\hline College & 187(38) & $57.38(3.60)$ \\
\hline$>$ College & $76(16)$ & $58.98(4.32)$ \\
\hline \multicolumn{3}{|l|}{ Income (RMB monthly) } \\
\hline$\leq 3000$ & $166(34)$ & $54.18(2.78)$ \\
\hline$>3000, \leq 6000$ & 213(44) & $55.96(4.33)$ \\
\hline$>6000$ & $107(22)$ & $59.81(3.67)$ \\
\hline \multicolumn{3}{|l|}{ Employment status } \\
\hline Fully employed & $420(86)$ & $56.28(3.68)$ \\
\hline Other & $66(14)$ & $55.69(5.86)$ \\
\hline \multicolumn{3}{|l|}{ Cancer Stage } \\
\hline I & $238(49)$ & $56.76(3.13)$ \\
\hline II & 167(34) & $55.48(4.21)$ \\
\hline III & $81(17)$ & $56.03(4.38)$ \\
\hline \multicolumn{3}{|l|}{ Surgery } \\
\hline Lumpectomy only & 287(59) & $56.16(4.24)$ \\
\hline Mastectomy only & $126(26)$ & $55.67(3.34)$ \\
\hline Mastectomy/reconstruction & $73(15)$ & $58.99(6.78)$ \\
\hline \multicolumn{3}{|l|}{ Radiation therapy } \\
\hline Yes & $397(82)$ & $56.35(3.48)$ \\
\hline No & $89(18)$ & $55.53(4.28)$ \\
\hline \multicolumn{3}{|l|}{ Chemotherapy } \\
\hline Yes & $338(70)$ & $56.27(3.86)$ \\
\hline No & $148(30)$ & $56.04(4.11)$ \\
\hline
\end{tabular}


Table 2: Association of demographic, clinical, and psychosocial characteristics with Benefit Finding scores ${ }^{\mathrm{a}}$

\begin{tabular}{|c|c|c|c|c|}
\hline \multirow[b]{2}{*}{ Characteristics } & \multicolumn{2}{|c|}{ Baseline model } & \multicolumn{2}{|c|}{ Longitudinal model $^{b}$} \\
\hline & $\beta(\mathbf{S E})$ & p-value & $\beta(\mathbf{S E})$ & p-value \\
\hline Age at Dx (per decade) & $-1.35(1.64)$ & 0.2622 & $-1.92(0.89)$ & 0.2212 \\
\hline Education & $1.68(0.56)$ & $0.0385^{*}$ & $1.79(0.76)$ & $0.0281^{*}$ \\
\hline Income (RMB/month) & $1.42(0.38)$ & $0.0475^{*}$ & $2.04(0.49)$ & $0.0388^{*}$ \\
\hline Stage & $-0.66(0.31)$ & 0.2675 & $-0.55(0.28)$ & 0.3672 \\
\hline Surgery & $0.84(0.21)$ & 0.1035 & $1.94(0.65)$ & 0.1286 \\
\hline Chemotherapy & $-0.34(0.11)$ & 0.0943 & $-0.53(0.15)$ & 0.1076 \\
\hline \multicolumn{5}{|l|}{ Social support } \\
\hline Baseline & $1.98(1.30)$ & $0.0021^{*}$ & $2.19(1.34)$ & $0.0009^{*}$ \\
\hline Change from baseline & & & $1.94(0.39)$ & $0.0003^{*}$ \\
\hline \multicolumn{5}{|c|}{ Spirituality (meaning and peace) } \\
\hline Baseline & $1.32(0.51)$ & $0.0004^{*}$ & $2.46(0.57)$ & $0.0002^{*}$ \\
\hline Change from baseline & & & $1.28(0.18)$ & $0.0001^{*}$ \\
\hline \multicolumn{5}{|l|}{ Disease coping } \\
\hline Baseline & $3.36(2.62)$ & $0.0001^{*}$ & $3.57(1.30)$ & $<0.0001^{*}$ \\
\hline Change from baseline & & & $3.26(0.43)$ & $<0.0001^{*}$ \\
\hline
\end{tabular}

${ }^{a}$ Demographic and medical variables that were nonsignificant in the models (marital status, employment status and radiation therapy) are not shown.

${ }^{\mathrm{b}}$ Longitudinal model using baseline value and change from baseline for time varying covariates.

${ }^{*} p \leq 0.05$. 
scoring, while a 1-point increase in disease coping predicted a 3.26-point increase. Education level and spirituality are also correlated with BFS changes, but to a lesser extent.

\subsection{Predictors of BFS Trends}

Table 3: Categorization of BFS trend patterns $(\mathrm{N}=486)$

\begin{tabular}{lrrr}
\hline Characteristics & $\mathbf{N}(\%)$ & Slope(M) & SD \\
\hline Entire population & $486(100)$ & -0.133 & 0.442 \\
$\beta \geq 0.3$ & $136(28)$ & 0.391 & 0.081 \\
$-0.3<\beta<0.3$ & $112(23)$ & -0.083 & 0.165 \\
$\beta \leq-0.3$ & $238(49)$ & -0.479 & 0.133 \\
\hline
\end{tabular}

Table 4: Predictors of BFS trend patterns by multivariate linear model $(\mathrm{N}=486)$

\begin{tabular}{lrr}
\hline Characteristics & $\beta($ SE) & p-value \\
\hline Age at Dx (per decade) & $-0.024(0.011)$ & 0.2310 \\
Education & $0.045(0.024)$ & 0.1004 \\
Income & $0.056(0.013)$ & 0.2684 \\
Cancer stage & $-0.048(0.011)$ & 0.2122 \\
Surgery & $0.084(0.036)$ & 0.1367 \\
Chemotherapy & $-0.028(0.010)$ & 0.1235 \\
Social support & $-0.032(0.017)$ & 0.1068 \\
Spirituality & $0.114(0.068)$ & $0.0289^{*}$ \\
Disease coping & $0.256(0.126)$ & $0.0004^{*}$ \\
\hline
\end{tabular}

The results of BFS trend analysis are presented in Table 3 It can be observed that the overall BFS of the sample decreases over time. In many participants, the first BF scoring after diagnosis (the baseline value) is the highest and it then generally decrease over time. Among all the participants, only $28 \%$ reported an upward trend over time $(\beta \geq 0.3)$, while around half (49\%) instead reported a downward trend $(\beta \leq-0.3)$; the remaining $23 \%$ reported no obvious change.

The results on association between the characteristics of the participants and BFS trends are presented in Table 4 . It can be observed that higher levels of spirituality and disease coping at baseline strongly indicated an increasing BFS trend $(p \leq 0.05)$. Specifically, among the participants with spirituality scoring above 80-points, the average slope of BFS trending is $0.266, \beta=0.266$, which is much higher than the average slope of the entire sample $(\beta=-0.133)$. The results w.r.t disease coping are similar. Among the participants with coping scoring above 80-points, the average slope coefficent of BFS trending is 0.438 , considerably higher than the average slope of the entire sample.

It is worthy to point out that some covariates of BFS such as education, income and social support are not reliable predictors of BFS trending pattern. In particular, higher social support scoring at baseline didn't not necessarily predict an upward BFS trend. Among the participants with the baseline social support above 80-point, the average slope of BFS trending is only -0.113 , which indicates no obvious change in BF scoring. Higher education and income similarly didn't indicate an upward trend either. Among the participants with at least Bachelor's degree, the average slope is only -0.102 . Among the participants with the monthly income above $6000 \mathrm{RMB}$, which can be considered high salary earners in western China, the value is similarly at -0.096 . None of them indicated an upward trend.

\section{Discussion}

Despite many negative physical and quality of life sequelae following breast cancer diagnosis and treatment, many patients report positive changes in their lives following cancer diagnosis. However, the existing studies focused on identifying the covariates of benefit finding among various demographic groups. The issue of how benefit finding 
evolves over time has not been adequately investigated. To our knowledge, this study is among the first to examine the trends of benefit finding evolution over time and their predictors in developing countries.

Consistent with previous studies conducted in American and European clinics [26, 27], our sample also reported considerable benefit findings among the participants after cancer. However, the observed degrees of benefit finding are however a little bit lower. This may result from the fact that the patients come from the north-western part of China, whose economy is less developed and advanced than the coastal areas of China, not to mention American and European areas. It is noteworthy that according to our study, benefit finding scoring at baseline is positively correlated with income level. This can to some extent explain less gains on BFS observed on our sample.

In the longitudinal association analysis, similar to what were observed in previous studies [28, 29, 2, 13], social support, spirituality and disease coping are closely correlated with changes of benefit finding scoring over time. It is noteworthy that education and income levels are associated with BF scoring as well. One probable explanation for this observation is that the patients with higher education and income levels have relatively richer social activities, thus receive more social support.

Interestingly, the results of trending model indicated that the average BFS in our sample actually decreased a little bit over time since diagnosis, at a rate of -0.133 per 6-month. Although it makes conceptual sense that benefit finding would increase over time following cancer diagnosis, our results indicated the opposite, which has also been reported in other demographic groups [13]. There are several probable explanations for this effect. First, the overall high levels of benefit finding at the time of cancer diagnosis may have resulted in a "ceiling effect" and regression to the mean over time. More importantly, the income levels of most patients in our sample were relatively low ( $<6000$ RMB per month). As time since diagnosis increases, survivors may struggle for bread and butter while cancer becomes a less salient stressor.

Most importantly, as shown in Table 4, spirituality and disease coping at diagnosis were positively related to benefit finding evolution over time. It can be observed that even though Han Chinese are mostly non-religious, life meaning and active coping are still critically important for sustaining benefit finding over time. Our results strongly indicated that regardless of demographics, purpose-oriented, or problem-solving rumination generally lead to higher levels of benefit finding, while aimless and passive way of life usually lead to lower levels. It is interesting to observe that the covariates of benefit findings may not reliably indicate its trending pattern. Specifically, the covariates of benefit finding observed in association analysis including education, income and social support can not accurately predict BFS trends. Even though higher education and income usually lead to higher initial levels of benefit finding, unfortunately they can not sustain its increase over time. Our data actually indicated the opposite: with all the participants with the income level above $6000 \mathrm{RMB}$ per month, the level of benefit finding decreases a little bit after the baseline measured at diagnosis. Among the participants with at least a Bachelor's degree, the level of benefit finding shows no obvious change after the baseline. In terms of social support, even though it is strongly correlated with benefit finding at almost all time points, higher level of social support at baseline may not sustain itself over time, thus does not necessarily indicate an increasing BFS trend.

\section{Conclusion}

From the data reported here, important results emerge. First, benefit finding usually develops very soon following a breast cancer diagnosis. However, it does not necessarily increase over time. On one hand, benefit finding may be supported by the salient threat of cancer recurrence, as threat and distress appear to be important aspects in the development of BF. But for patients in less developed areas with limited income, the threat may become a less salient stressor for survivors over time. Secondly, our study showed that even though benefit findings may decrease over time, the efforts of meaning making and active coping can effectively counter its negative trend. Uncovering the underlying factors sustaining positive BFS changes in the experience of cancer is the first step to increase long-term post-traumatic growth of breast cancer patients.

However, this study had several limitations, which need to be further investigated in future work. First, the sample has limited diversity, i.e. participants consist of mostly non-religious Han Chinese women, which may limit generalizability. Future studies should consider recruiting a larger sample to replicate these findings in a minority Chinese population. Secondly, our study focused on the benefit finding immediately after diagnosis. Predictors of BFS trends were estimated based on variable measurements at baseline. The psychosocial characteristics of cancer 
patients may vary over time. Therefore, it may be necessary to increase the assessment window and investigate how long-term post-traumatic growth evolves long after diagnosis.

\section{Declarations}

\subsection{Ethics approval and consent to participate}

The experimental protocol was established, according to the ethical guidelines of the Helsinki Declaration and was approved by the Research Ethics Committee of Xijing Hospital, the Air Force Military Medical University. Written informed consent was obtained from individual or guardian participants.

\subsection{Consent for publication}

Not applicable.

\subsection{Availability of data and material}

The datasets generated during and/or analysed during the current study are not publicly available due to the privacy policy concerning private information of active army personnel, but are available from the corresponding author on reasonable request.

\subsection{Competing interests}

The authors declare that they have no competing interests.

\subsection{Funding}

The study has been supported by National Natural Science Foundation of China (grant No. 81671910 and 81572816) and the Air Force Military Medical University (grant No. XJZT18MDT09).

\subsection{Authors' contributions}

W.Y Bi was the chief designer of the questionnaire, collected and pre-processed the data of participants, and was the chief writer of the introduction and methods sections. H.N Wang initiated and supervised the project, was also the chief writer of the discussion section. C.L Zhu was the chief designer of the analysis strategies, conducted technical analysis, and was also the chief writer of the results section. G.Y Yang was the co-writer of the results and discussion section. All authors read and approved the final manuscript.

\subsection{Acknowledgements}

Not applicable.

\section{References}

[1] P. Tomich, V. Helgeson, Is finding something good in the bad always good? benefit finding among women with breast cancer, Health Psychol 23 (1) (2004) 16-23.

[2] C. Conley, B. Andersen, Lemons to lemonade: effects of a biobehavioral intervention for cancer patients on later life changes, Health Psychol 38 (3) (2019) 206-216.

[3] R. Tedeschi, L. Calhoun, Posttraumatic growth: conceptual foundations and empirical evidence, Psycho Inquiry 15 (1) (2004) 1-18.

[4] L. Jansen, M. Hoffmeister, J. Chang-Claude, H. Brenner, V. Arndt, Benefit finding and post-traumatic growth in long-term colorectal cancer survivors: prevalence, determinants, and associations with quality of life, Br J Cancer 105 (8) (2011) 1158-1165.

[5] N. Koutrouli, F. Anagnostopoulos, G. Potamianos, Posttraumatic stress disorder and posttraumatic growth in breast cancer patients: a systematic review, Women Health 52 (5) (2012) 503-516.

[6] C. RL, T. SE, S. LA, A better world or a shattered vision? changes in life perspective following victimization, Social Cognition 8 (1990) $263-285$.

[7] H. VS, R. KA, T. PL, A meta-analytic review of benefit finding and growth, J Consult Clin Psychol 74 (5) (2006) $797-816$.

[8] B. J, M. MH, H. V, C. PR, S. CM, The posttraumatic growth inventory: an examination of the factor structure and invariance among breast cancer survivors, Psycho-oncology 19 (8) (2010) 830-838.

[9] A. MH, L. JM, K. KM, et al., Cognitive-behavioral stress management intervention decreases the prevalence of depression and enhances benefit finding among women under treatment for early-stage breast cancer, Health Psychol 20 (1) (2001) 20-32. 
[10] B. KM, M. MF, A. NK, R. JH, Positive and negative life changes experienced by survivors of non-hodgkin's lymphoma, Ann Behav Med 34 (2) (2007) 188-199.

[11] C. ME, L. Joseph, M. Alyson, et al., Contemplative self healing in women breast cancer survivors: a pilot study in underserved minority women shows improvement in quality of life and reduced stress, BMC Complementary and Alternative Medicine 14 (2014) 349.

[12] T. T, P. M, K. M, et.al, Perceived social support in african american breast cancer patients: predictors and effects, Soc Sci Med 192 (2017) $134-142$.

[13] C. CC, S. BJ, C. J, et al., Patterns and covariates of benefit finding in young black breast cancer survivors: A longitudinal, observational study., Psycho-Oncology 29 (2020) 1115-1122.

[14] S. SR, S. AL, D.-B. S, The yellow brick road and the emerald city: benefit finding, positive reappraisal coping and post-traumatic growth in women with early-stage breast cancer, Health Psychol 22 (5) (2003) 487-497.

[15] W. T, Correlates of posttraumatic growth in married breast cancer survivors, J Soc Clin Psychol 23 (5) (2004) $733-746$.

[16] B. JE, M. BE, B. CA, et al., Perceptions of positive meaning and vulnerability following breast cancer: predictors and outcomes among long-term breast cancer survivors, Ann Behav Med 29 (3) (2005) 236-245.

[17] M. F, V. AJ, C. JW, van de Poll-Franse LV, Well-being, posttraumatic growth and benefit finding in long-term breast cancer survivors, Psychol Health 24 (5) (2009) 583-595

[18] S. EC, O. C, B. I, Posttraumatic growth in cancer:reality or illusion?, Clin Psychol Rev 29 (1) (2009) $24-33$.

[19] S. CD, S. AL, The mos social support survey, Soc Sci Med 32 (1991) 705-714.

35 [20] H. JS, K. RL, M. JD, W. D, Measures and concepts of social support, Social Support and Health 98 (1985) 83-108.

[21] C. DF, T. DS, G. G, et al, The functional assessment of cancer therapy scale: development and validation of the general measure, J Clin Oncol 11 (1993) 570-579.

[22] P. AH, F. G, B. MJ, H. L, C. D, Measuring spiritual well-being in people with cancer: the functional assessment of chronic illness therapy spiritual well-being scale (facit-sp), Ann Behav Med (2002) 49-58.

40 [23] C. C, You want to measure coping but your protocol's too long: consider the brief cope, Int J Behav Med 4 (1) (1997) 92-100.

[24] H. H, H. J, Z. T, et al, Reliability and validity of the benefit finding scale for breast cancer patients, Chin J Prac Nurs 30 (33) (2014) $27-29$.

[25] A. MH, L. JM, K. KM, et al, Cognitive-behavioral stress management intervention decreases the prevalence of depression and enhances benefit finding among women under treatment for early-stage breast cancer, Health Psychol 20 (1) (2001) $20-32$.

[26] T. PL, H. VS, Is finding something good in the bad always good? benefit finding among women with breast cancer, Psychol Health 23 (1) (2004) 16-23.

[27] U. KR, B. AE, C. CS, A. MH, Finding benefit in breast cancer: relations with personality, coping, and concurrent well-being, Psychol Health 20 (2) (2005) 175-192.

[28] H. CL, W. TA, L. MS, S. P, J. SE, S. EK, A comparison of a spiritually based and non-spiritually based educational intervention for informed decision making for prostate cancer screening among church-attending african-american men, Urol Nurs 29 (4) (2009) $249-258$.

[29] C. MW, H. SM, T. RG, L. CW, The valence of attentional bias and cancer-related rumination in posttraumatic stress and posttraumatic growth among women with breast cancer, Psycho-oncology 20 (5) (2011) 544-552. 